\title{
Stage IVA Thymoma AJCC v8
}

National Cancer Institute

\section{Source}

National Cancer Institute. Stage IVA Thymoma AICC v8. NCI Thesaurus. Code C136354.

Stage IVA includes: (Any T, N1, M0); (Any T, N0,1, M1a). N0: No regional lymph node metastasis. N1: Metastasis in anterior (perithymic) lymph nodes. M0: No distant metastasis. M1a: Separate pleural or pericardial nodule(s). (AJCC 8th ed.) 